This item was submitted to Loughborough's Research Repository by the author.

Items in Figshare are protected by copyright, with all rights reserved, unless otherwise indicated.

\title{
Optimisation based control framework for autonomous vehicles: algorithm and experiment
}

PLEASE CITE THE PUBLISHED VERSION

http://dx.doi.org/10.1109/ICMA.2010.5588100

PUBLISHER

(C) IEEE

VERSION

VoR (Version of Record)

LICENCE

CC BY-NC-ND 4.0

\section{REPOSITORY RECORD}

Liu, Cunjia, Wen-Hua Chen, and J.D. Andrews. 2011. "Optimisation Based Control Framework for Autonomous Vehicles: Algorithm and Experiment”. figshare. https://hdl.handle.net/2134/7731. 
This item was submitted to Loughborough's Institutional Repository (https://dspace.lboro.ac.uk/) by the author and is made available under the following Creative Commons Licence conditions.

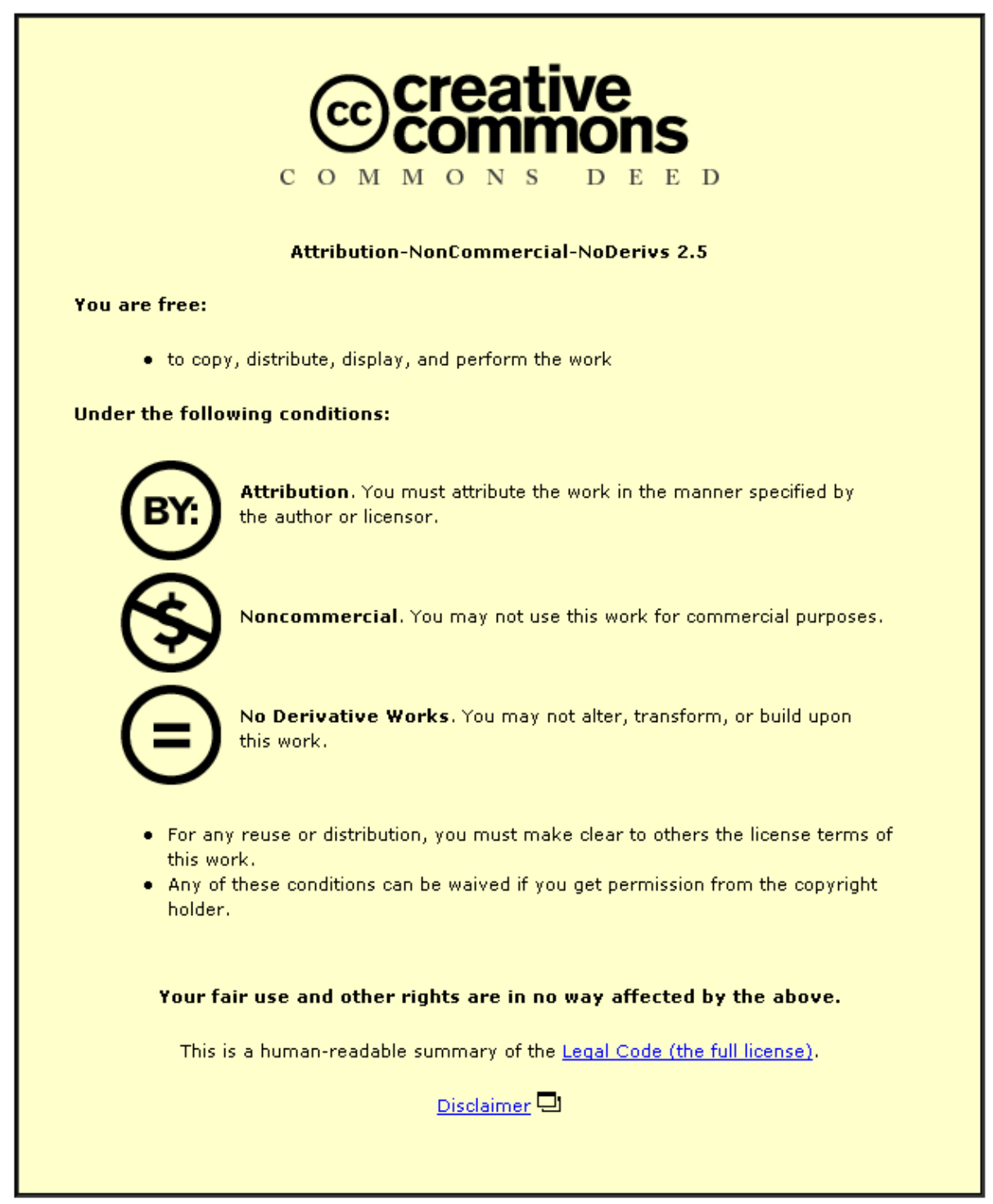

For the full text of this licence, please go to: http://creativecommons.org/licenses/by-nc-nd/2.5/ 


\title{
Optimisation based control framework for autonomous vehicles: algorithm and experiment
}

\author{
Cunjia Liu, Wen-Hua Chen, Senior Member, IEEE, John Andrews
}

\begin{abstract}
This paper addresses both path tracking and local trajectory generation for autonomous ground vehicles. An optimisation based two-level control framework is proposed for this task. The high-level control operates in a receding horizon fashion by taking into account real-time sensory information. It generates a feasible trajectory satisfying the nonlinear vehicle model and various constraints, and resolves possible short term conflicts through on-line optimisation. The low-level controller drives the vehicle tracking the local trajectory in the presence of uncertainty and disturbance. It is shown that the time varying controller proposed in this paper guarantees stability under all possible trajectories. The two-level control structure significantly facilitates the real-time implementation of optimisation based control techniques on systems with fast dynamics such as autonomous vehicle systems. The proposed technique is implemented on a small-scale autonomous vehicle in the lab. Both simulation and experimental results demonstrate the efficiency of the proposed technique.
\end{abstract}

\section{INTRODUCTION}

Autonomous vehicles, including unmanned aerial vehicles (UAV) and ground vehicles (UGV), have been drawing a considerable attention from both industry and academic in the past two decades, with the expectation of a wide range of applications. Versatile tasks can be performed by autonomous vehicles to replace human or human operated vehicles. An essential function required for an autonomous vehicle performing a mission is to track reference trajectories or waypoints either pre-planned beforehand or dynamically generated by a supervisory layer such as human operators or intelligent mission planner.

The mission-level planner for autonomous vehicles focuses on achieving the global goal while minimising risk and operates at a large time scale. In this setting, the vehicle is usually treated as a point mass and routines are generated mainly to maximize the mission success rate by taking into account the location of targets, threats and geographic information [1]. Hence trajectories generated by the high-level mission planner may not fully satisfy vehicle kinematic constraints and may also need alterations when encountering newly developed threats or obstacles.

To this end, local trajectory generation and vehicle control has been studied by many researchers and a number of methods have been proposed [2]. As all the autonomous

Cunjia Liu and Wen-Hua Chen are with Department of Aeronautical and Automotive Engineering, Loughborough University, Loughborough, LE11 3TU, UK. e-mail: \{c.liu5, w.chen $\} @ 1 b o r o . a c . u k$

John Andrews is with Nottingham Transportation Engineering Centre, University of Nottingham, Nottingham, NG7 2RD, UK. e-mail: John.Andrews@nottingham.ac.uk vehicles are equipped with sensors that can provide local information within a certain range, model predictive control (MPC) provides a promising, natural framework for this problem since the environment information is changed/updated as the vehicle proceeds. Both vehicle dynamics and environmental information can be considered in this approach. Therefore it is argued that MPC may offer a number of advantages over other methods [3].

The vehicle under consideration in this paper is a rearwheel-driven ground vehicle. The kinematics of such a vehicle is commonly described by a nonholonomic nonlinear model. Applications of MPC on nonholonomic mobile robots can be found in [4], [5]. The corresponding MPC stability issues also have been discussed in [6], [7]. More recently, a UAV path following problem has been cast into this category and solved using MPC, but the obstacle avoidance has not been taken into account [8].

In MPC setting, an optimisation problem (OP) has to be solved within each sampling interval. This is the main obstacle of applying MPC into plants with fast dynamics such as vehicles. Although a few MPC algorithms in this field have been implemented in real-time [9], [10], they are based on linear MPC settings. For nonlinear vehicle models with obstacles avoidance function, a computationally intensive nonlinear optimisation problem is involved in calculation of the control command. Real-time implementation of nonlinear MPC on autonomous vehicles still poses a major challenge [11].

This paper proposes an optimisation based control framework which combines MPC with traditional control techniques. Instead of attempting to implement a single nonlinear MPC, the proposed framework employs a two-level control structure where the high-level controller generates local trajectories by exploiting both vehicle and environment information updated by sensors in real-time such as obstacles and other road users. The vehicle information includes a nonlinear vehicle model and various constraints such as nonholonomic constraints and maximum control inputs. The lowlevel controller is designed based on the linearisation around a reference trajectory provided by the high-level controller to stabilise the vehicle in the presence of disturbances and uncertainties. These two levels of controller are operated at different time scales: the high-level on a lower sampling rate, whereas the low-level on a higher one. Therefore the proposed optimisation based two-level control framework facilitates real-time implementation since it can simultaneously cope with the heavy computational burden demanded by on-line 
optimisation solvers and fast feedback as required by vehicle stabilization and control. Moreover, the high-level MPC adopts a modified formulation to further reduce the computation load comparing to the early similar works [12].

With the feature of reduced computational requirements on the real-time implementation, the functions of proposed control framework include:

- regenerating feasible local trajectories for the vehicles satisfying various constraints such as nonholonomic constraints and maximum control inputs and nonlinear vehicle models;

- resolving short term conflicts with other road users and obstacles;

- tracking the generated trajectories in the presence of disturbances and uncertainties with a local controller that guarantees stability under all possible trajectories.

The rest of this paper is organised as follows. Section II describes the high-level MPC formulation in a discrete time setting with an emphasis on the real-time implementation. The two-level framework including the design of low-level controller is introduced in Section III. In Section IV the simulation and experiment results are illustrated to verify the proposed approach, followed by a conclusion in Section V.

\section{MPC FORMULATION}

\section{A. Vehicle model}

Despite the apparent simplicity of the kinematic model of a car-like vehicle, the design of controller for this kind of system can be considered a challenge owing to the existence of nonholonomic constraints. Basically, the nonholonomic constraint related to a car-like vehicle refers to the constraint of rolling without slipping between the rear wheels and ground.

The configuration of the rear-wheel driving vehicle, as the TT01 radio controlled model car used in our lab, is shown in Fig. 1.
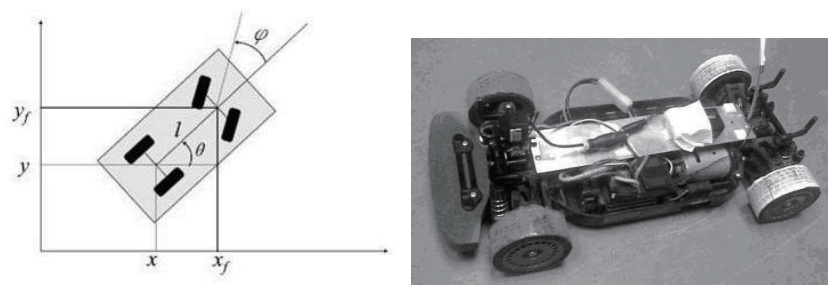

Fig. 1. Vehicle configuration

The states of the model are $\mathbf{x}=\left[\begin{array}{lll}x & y & \theta\end{array}\right]^{\prime}$, where $(x, y)$ are the coordinates of the centre point of the rear axle, $\theta$ is the heading angle of the car body with respect to the $x$ axis, and $\varphi$ is the steering angle of the front wheel with respect to the vehicle's longitudinal line, which can be seen as a control input. Another parameter of the model is the distance between the front axle and the rear axle, which is $l$ in the Fig. 1. The kinematical relationship can be described using the following mathematical model:

$$
\begin{aligned}
& \dot{x}=v \cdot \cos \theta \\
& \dot{y}=v \cdot \sin \theta \\
& \dot{\theta}=v \cdot \frac{\tan \varphi}{l}=v \cdot c
\end{aligned}
$$

where the control inputs for the vehicle are: $\varphi$ the steering angle and $v$ the line velocity. The curvature can be defined as:

$$
c=\tan \varphi / l
$$

For simplicity of control design, $c$ is used as an input. That is, $\mathbf{u}=\left[\begin{array}{ll}c & v\end{array}\right]^{\prime}$. If the input $c$ is calculated, the steering angle $\varphi$ can be derived from the relationship (2). The nonholonomic constraint can be represented as follows:

$$
\dot{x} \sin (\theta+\varphi)-\dot{y} \cos (\theta+\varphi)-\dot{\theta} \cos \theta \cdot l=0
$$

One can easily verify that the nonholonomic constraint (3) is involved in this model. In terms of the control constraints, the steering angle is usually restricted within the range imposed by the actual mechanical saturation. The steering angle limit also imposes a minimum turning radius on the vehicle, which is:

$$
R_{\min }=\left|c_{\max }\right|^{-1}=l / \tan \varphi_{\max }
$$

The constraints on the car velocity can also be added in the same way.

\section{B. MPC algorithm}

Obviously, the kinematic constraint and the input constraints prohibit the vehicle from tracking arbitrary trajectories generated by the mission level planner where the vehicle is usually considered as a point mass and some constraints have not been taken into account. Therefore, the tracking controller needs to address both local trajectory regeneration and tracking problems, i.e., generating the optimal, at least a feasible, trajectory and the corresponding control sequence based on a given reference trajectory or a set of way points.

MPC is an optimal control strategy that uses the model of the plant to obtain the optimal control sequence by minimizing an objective function. At each sampling instant, a model is used to predict the behaviour of the plant over a prediction horizon [13]. Based on these predictions, the objective function is minimized with respect to the future sequence of input. Thus MPC requires solving a constrained OP for each sampling instant. Although prediction and optimisation are performed over a future time horizon, only the control inputs for the current sampling step or first few steps are eventually used to drive the system. The same procedure is repeated at the next sampling instant with updated system states for a receding horizon.

Since the MPC algorithm is implemented on a digital computer, a difference equation is required and usually approximated form differential equation (1) by using Euler or other approximations with $T_{d}$ as the discretization parameter. There is an error between the discrete model and continuous model which monotonically increases with $T_{d}$. The MPC designed on the discrete model can stabilise the original continuous model 
if $T_{d}$ is small enough [14]. However, the small $T_{d}$ increases the computational burden as there are more control variables to be decided within the same prediction duration. If solving OP cannot be completed in such a time interval, MPC cannot be implemented in real-time. This is particularly important for safety critical systems with fast dynamics such as autonomous vehicles.

To avoid this problem, this paper introduces another important parameter, namely the MPC sampling time $T_{s}$, defined as the interval of the MPC updating the current states and generating the new control sequence. In the conventional MPC setting, $T_{d}=T_{s}$. However, with respect to $T_{d}$ and $T_{s}$ we define the control holding horizon $N$, which indicates the control inputs keeping the constant values for $N$ integration steps. These three parameters have to satisfy the relationship: $T_{s}=N \cdot T_{d}$. With the modified time setting the time allowed for OP solving is increased to $T_{s}$, while maintaining the resolution of the integration to $T_{d}$ in the prediction. Note that although other MPC formulations may also have the different sampling time like $T_{s}$ and $T_{d}$ [8], [12], they did not use the control holding mechanism.

To clearly explain the timing setting, an example is illustrated in Fig 2. The control holding horizon $N$ is set to 4 steps, same with the prediction horizon $H$. Within the period of $T_{s}$ the control variables are set to constant, while the integration of system equation follows the discrete sampling time $T_{d}$. This setting maintains the accuracy of the prediction but significantly reduces the number of optimisation variables covering the same length of prediction.

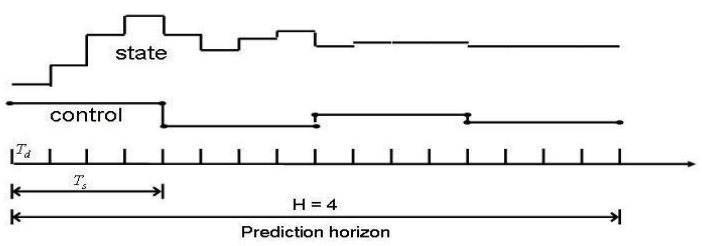

Fig. 2. Time setting example

Under this setting, a discrete MPC is employed for tracking control. By defining the reference trajectory as $\mathbf{x}_{r}$ and the tracking error $\mathbf{x}_{e}=\mathbf{x}_{r}-\mathbf{x}$, we can formulate the following objective function to be minimised:

$$
\begin{aligned}
& J(k)=F(\mathbf{x}(k+H N \mid k))+ \\
& \sum_{i=0}^{H-1} \sum_{j=0}^{N-1} L(\mathbf{x}(k+i N+j \mid k), \mathbf{u}(k+i n+j \mid k)) \\
& L\left(\mathbf{x}(k), \mathbf{x}_{r}(k), \mathbf{u}(k)\right)=\mathbf{x}_{e}(k)^{\prime} Q \mathbf{x}_{e}(k)+\mathbf{u}(k)^{\prime} R \mathbf{u}(k) \\
& F\left(\mathbf{x}(k+H N), \mathbf{x}_{r}(k)\right)=\mathbf{x}_{e}(k+H N)^{\prime} P \mathbf{x}_{e}(k+H N)
\end{aligned}
$$

where, $L\left(\mathbf{x}(k), \mathbf{x}_{r}(k), \mathbf{u}(k)\right)$ is the penalty for each time step, $F\left(\mathbf{x}(k+H N), \mathbf{x}_{r}(k)\right)$ is the terminal penalty, $H$ is the prediction horizon, $N$ is the control holding horizon, $P, Q$ and $R$ are the positive definite weighting matrices.
The control holding horizon plays an important role in the modified MPC formulation. If $N=1$ the modified MPC reverts to the conventional MPC setting. For a given prediction duration required by the closed-loop stability, increase of $N$ can reduce the number of variables to be optimased. In contrast, for a given number of optimisation variables that the online solver can handle, increase of $N$ can expand the prediction length.

The nonlinear optimization problem that minimises the objective function subjected to various constraints can be stated as:

$$
\mathbf{x}_{r e f}, \mathbf{u}_{r e f}=\arg \min _{\mathbf{x}, \mathbf{u}} J(k)
$$

subject to:

$$
\begin{aligned}
& \mathbf{x}(k+j+1 \mid k)=f(\mathbf{x}(k+j \mid k), \mathbf{u}(k+j \mid k)) \\
& \mathbf{x}(k+j \mid k) \in \mathbf{X} \\
& \mathbf{u}(k+j \mid k) \in \mathbf{U} \\
& j=0,2, \cdots, N-1 \\
& \mathbf{x}(k \mid k)=\mathbf{x}_{0}
\end{aligned}
$$

where the first term is the compact form of vehicle dynamics, and $\mathbf{X}, \mathbf{U}$ are control and state constraints, respectively. This optimization problem must be solved at each sampling instant, producing the local reference trajectory $\mathbf{x}_{r e f}$, and the optimal control sequence $\mathbf{u}_{r e f}$. The related MPC stability issue has been discussed in [6] by imposing a terminal constraint, however it can be omitted in the implementation if the prediction is sufficiently long [13].

\section{Obstacle avoidance}

Autonomous vehicles operating in an unknown and unstructured environment may encounter obstacles not being taken into account in the mission-level path planning and other road users such as other manned and unmanned vehicles. Collision avoidance is important for resolving short term conflicts while following reference trajectories.

By adding a potential function into the cost function of the OP, the MPC based framework is endowed with the obstacle avoidance ability [15]. Assume that the obstacle has a round shape with a radius of $R_{o}$, and its position $\left(x_{o}, y_{o}\right)$ can be detected by vehicle sensors. The obstacle avoidance term in cost function can be defined in (7):

$$
J_{o}=\frac{k_{o}}{\sqrt{\left(x-x_{o}\right)^{2}+\left(y-y_{o}\right)^{2}}-R_{o}+\varepsilon}
$$

where $\sqrt{\left(x-x_{o}\right)^{2}+\left(y-y_{o}\right)^{2}}>R_{o}, k_{o}$ is the weighting parameter, and $\varepsilon$ is a small positive constant for non-singularity. The obstacle term (7) is added into the cost function (5) in the formulated optimisation problem (6). In this paper, the obstacle position is assumed to be known by the vehicle. In reality, this can be achieved by using onboard laser senor.

\section{Two-LEVEl CONTROL Framework}

The proposed MPC scheme eases the computational burden by (i) increasing the computational interval to give more time for optimisation and (ii) significantly reducing the variables to 
be optimised. However, the MPC strategy becomes an openloop optimal control within the interval $T_{s}$. Unfortunately, due to the mismatch between the mathematical model and the real plant, the noises and disturbances in the process, this kind of optimal control would not perform as being designed. Within the interval $T_{s}$, the MPC cannot suppress any tracking error. The bandwidth associated with the MPC is not adequate for stabilising and control the vehicles that have fast dynamics.

In order to avoid these difficulties in the MPC, a twolevel structure is adopted in the control framework. The highlevel controller is the discrete MPC strategy described before, which can provide optimised reference trajectory and the corresponding control inputs, whereas the low-level controller is a conventional feedback controller that can track the local trajectories and provide stability around the trajectory in the presence of disturbances and uncertainties. The high-level controller runs at a lower sampling rate $T_{s}$ to adapt the calculation time caused by solving the nonlinear optimisation problem. In contrast, the low-level controller works at a much higher sampling rate to stabilise the plant. The control structure is shown in Fig 3.

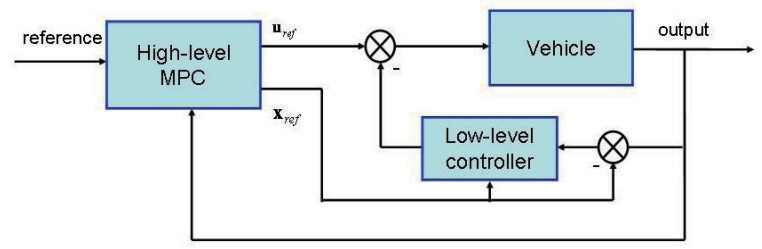

Fig. 3. Two-level control framework

In the implementation, the high-level MPC provides optimal local trajectories and also the corresponding optimal control inputs. The low-level controller measures current states and then compares them with the high-level state reference. The error signals are used to generate compensation control efforts through the local controller. The overall control inputs applied to the vehicle consist of two parts: the reference control inputs and the compensation control generated by the local controller.

The low-level controller can be designed based on perturbation models around the reference state produced by high-level MPC. Since the low-level control works in a much higher sampling rate, the controller design can be performed in the continuous time domain. The vehicle model (1) can be linearised around the trajectory and nominal inputs $\left(\mathbf{x}_{r e f}, \mathbf{u}_{r e f}\right)$ as following:

$$
\begin{aligned}
\dot{\mathbf{x}}=f(\mathbf{x}, \mathbf{u}) \approx f\left(\mathbf{x}_{r e f}, \mathbf{u}_{r e f}\right) & +\left.\frac{\partial f}{\partial x}\right|_{\mathbf{x}_{r e f}}\left(\mathbf{x}-\mathbf{x}_{r e f}\right) \\
& +\left.\frac{\partial f}{\partial u}\right|_{\mathbf{u}_{r e f}}\left(\mathbf{u}-\mathbf{u}_{r e f}\right)
\end{aligned}
$$

By defining the error state $\Delta \mathbf{x}=\mathbf{x}-\mathbf{x}_{r e f}$ and control compensation $\Delta \mathbf{u}=\mathbf{u}-\mathbf{u}_{r e f}$. The error system can be stated as:

$$
\Delta \dot{\mathbf{x}}=\left.\frac{\partial f}{\partial x}\right|_{\mathbf{x}_{r e f}} \Delta \mathbf{x}+\left.\frac{\partial f}{\partial u}\right|_{\mathbf{u}_{r e f}} \Delta \mathbf{u}=A \Delta \mathbf{x}+B \Delta \mathbf{u}
$$

where

$$
A=\left[\begin{array}{ccc}
0 & 0 & -v_{r e f} \cdot \sin \theta_{r e f} \\
0 & 0 & v_{r e f} \cdot \cos \theta_{r e f} \\
0 & 0 & 0
\end{array}\right] B=\left[\begin{array}{cc}
0 & \cos \theta_{r e f} \\
0 & \sin \theta_{r e f} \\
v_{r e f} & c_{r e f}
\end{array}\right] .
$$

In terms of the time varying system (9), a time varying feedback law is proposed as in (10):

$$
K=\left[\begin{array}{ccc}
-k_{1} \sin \theta_{\text {ref }} & k_{1} \cos \theta_{\text {ref }} & k_{2} \\
k_{3} v_{\text {ref }} \cos \theta_{\text {ref }} & k_{3} v_{\text {ref }} \sin \theta_{\text {ref }} & 0
\end{array}\right]
$$

where $k_{1}, k_{2}$ and $k_{3}$ are control parameters to be tuned, the other parameters depend on the high-level reference. Then the state transition matrix of the closed-loop error system $\Delta \dot{\mathrm{x}}=$ $(A-B K) \Delta \mathbf{x}$ is derived in (11):

$$
v \cdot\left[\begin{array}{ccc}
-k_{3} \cos ^{2} \theta & -k_{3} \cos \theta \sin \theta & -\sin \theta \\
-k_{3} \cos \theta \sin \theta & -k_{3} \sin ^{2} \theta & \cos \theta \\
k_{1} \sin \theta-c k_{3} \cos \theta & -k_{1} \cos \theta-c k_{3} \sin \theta & -k_{2}
\end{array}\right]
$$

For the sake of simplicity, the subscripts for the reference states are omitted. Since the resulting close-loop system is a linear time-varying system, the stability can be analysed by using Lyapunov theory.

By defining the Lyapunov function as (12), and invoking the closed-loop system (11), the derivative of the Lyapunov function is derived in (13).

$$
\begin{gathered}
V(\Delta \mathbf{x})=0.5\left(\Delta x^{2}+\Delta y^{2}+\Delta \theta^{2}\right) \\
\dot{V}=-v \cdot k_{3}(\Delta x \cos \theta+\Delta y \sin \theta)^{2}-v \cdot k_{2} \Delta \theta^{2} \\
-v c \cdot k_{3}(\Delta x \cos \theta+\Delta y \sin \theta) \Delta \theta \\
v \cdot\left(1-k_{1}\right)(-\Delta x \sin \theta+\Delta y \cos \theta) \Delta \theta
\end{gathered}
$$

By defining the tracking error in the vehicle body coordinates: $\Delta x_{b}=\Delta x \cos \theta+\Delta y \sin \theta, \Delta y_{b}=-\Delta x \sin \theta+\Delta y \cos \theta$ and $\Delta \theta_{b}=\Delta \theta$, the equation (13) is equivalent to (14).

$$
\begin{aligned}
\dot{V}= & -v k_{3}\left(\Delta x_{b}-\frac{c}{2} \Delta \theta\right)^{2}-v\left(k_{2}-\frac{c^{2}}{2} k_{3}\right) \Delta \theta^{2} \\
& -v\left(k_{1}-1\right) \Delta y_{b} \Delta \theta_{b}
\end{aligned}
$$

To guarantee the stability of closed-loop, i.e. $\dot{V}<0$, the design of low-level control parameters has to follow the rules in (15):

$$
\begin{aligned}
& k_{3}>0 \\
& k_{2}>\frac{c_{\text {max }}^{2}}{2} k_{3} \\
& \left(k_{1}-1\right) \operatorname{sign}\left(\Delta y_{b} \Delta \theta_{b}\right)>0
\end{aligned}
$$




\section{Simulation AND EXPERIMENT}

\section{A. Numerical simulation}

To show the performance of the MPC strategy, simulation was firstly carried out based on the Subaru TT01 model car in the lab. After various driving tests in the lab, the model of the vehicle has been built. Simulation for a number of tasks has been performed.

One simulation task is to track an eight-shaped trajectory with obstacles. In the simulation, the matrices $Q$ and $R$ in the cost function of MPC are chosen as $\operatorname{diag}(1,1,0.5)$ and $\operatorname{diag}(0.1,0.1)$ respectively. The terminal penalty weighting $P$ is chosen as $\operatorname{diag}(10,10,5)$. The discretization time of model $T_{d}$ is $0.1 s$ and the MPC sampling time $T_{s}$ is set to $0.5 s$ which means control holding horizon $N$ is 5 steps. The prediction horizon $H$ is set to 6 steps, suggesting that the overall prediction duration is 3 seconds. In terms of input constraints, the steering angle $\varphi$ is limited from $-0.4 \mathrm{rad}$ to $0.4 \mathrm{rad}$ and the line speed $v$ from $0.15 \mathrm{~m} / \mathrm{s}$ to $0.8 \mathrm{~m} / \mathrm{s}$. The low-level control parameters are $k_{1}=1 \pm 0.5$ depending on $\operatorname{sign}\left(\Delta y_{b} \Delta \theta_{b}\right), k_{2}=2.5$ and $k_{3}=1$.

The simulation results are shown in Fig 4. It can be seen that although the initial position (square marker) of the vehicle is not on the reference trajectory, the controller drives the vehicle back to the reference track and all obstacles are avoided. The average OP solving time is less than 0.05 second using a computer with $2.3 \mathrm{GHz} \mathrm{CPU}$, however the maximum time can reach to 0.4 second when the measured error states is far away from the reference at the beginning or encounters an obstacle. The control signals are also shown in Fig 4.
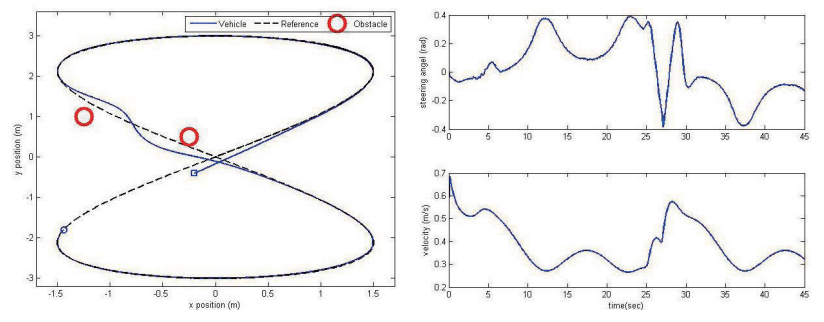

Fig. 4. Eight-shape tracking with obstacles

In terms of car-like vehicles, the kinematic constraints and the input constraints prohibit the vehicle from tracking arbitrary trajectories. However, in some applications of autonomous vehicle, there is no chance to design an elaborate trajectory beforehand. The high-level operator may just provide trajectory connected by straight lines and curves or some waypoints. Within one prediction horizon, the MPC strategy can find a feasible trajectory closing to the reference trajectory or waypoints, as well as the corresponding control sequence.

Performance of tracking arbitrary trajectory is demonstrated by simulations. The task is forcing the vehicle following a square trajectory at a constant speed which by no means can be tracked by a car-like vehicle accurately as nonholonomic constraints and minimum turning radius are not satisfied. How- ever, the optimisation based control framework can provide an optimised smooth trajectory to follow.

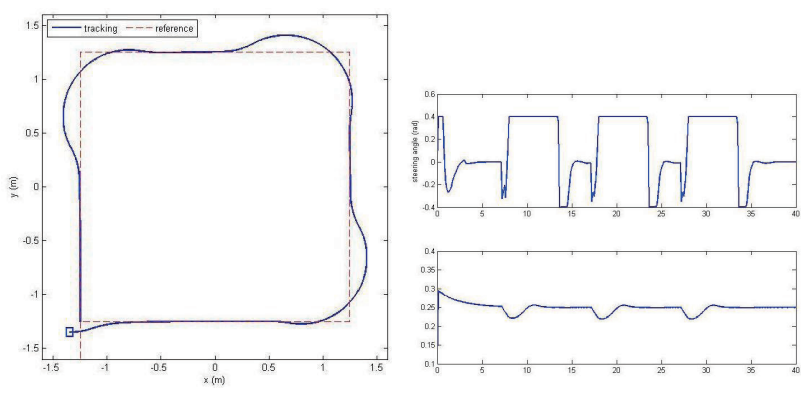

Fig. 5. Square trajectory tracking

The simulation results can be found in Fig (5). The tracking trajectory is smooth and the constraints for both steering angle and speed are respected. Parameter settings are as same as the previous simulation. It can be seen that at the corner of the trajectory, the speed of the car is reduced to get a better turning as a human driver does. The big error at each corner is due to the minimum turning radius of the vehicle. For the vehicle with $l=0.25 \mathrm{~m}$ and maximum steering angle $0.4 \mathrm{rad}$ the minimum turning radius based on (4) is about $0.6 \mathrm{~m}$. The computation load of this optimisation problem is heavier than the previous one. This is because there is no input reference for the corresponding trajectory, thereby the initial guess of the optimisation variables are much different form their optimal values, especially when vehicle turning around the corners. The maximum of solving time during the simulation is approaching $0.5 \mathrm{~s}$.

\section{B. Experiment setup and result}

The implementation of the two-level control framework in real-time is achieved by integrating Matlab and its XPC target real-time environment. The low-level controller is located on xPC target, and the high-level controller is executed on another computer in the Matlab environment using fmincon function to solve the optimization problem online. The information exchange between them relies on the local area network (LAN) with UDP protocol (Fig 6). The synchronization between the two computers is guaranteed by the real-time xPC target application calling Matlab program based on its own timer. The xPC target also integrates interfaces to the sensors (Vicon Motion Capture system) and radio controller which measure the vehicle states and send control signals, respectively.

In the experiments the low-level control operates at $50 \mathrm{~Hz}$, and the high-level MPC updating interval $T_{s}$ is $0.5 \mathrm{~s}$. The other parameters are kept as the same as in simulations. The experiment result of tracking with obstacle avoidance is shown in Fig 7, where there is one obstacle staying on the reference trajectory. Due to the limitation of the testing area, only half eight-shape reference can be tracked. The corresponding control inputs are shown in Fig 7 as well.

The experiment result of tracking a square trajectory (shown by 'triangles') is shown in Fig 8. The dots in the figure 


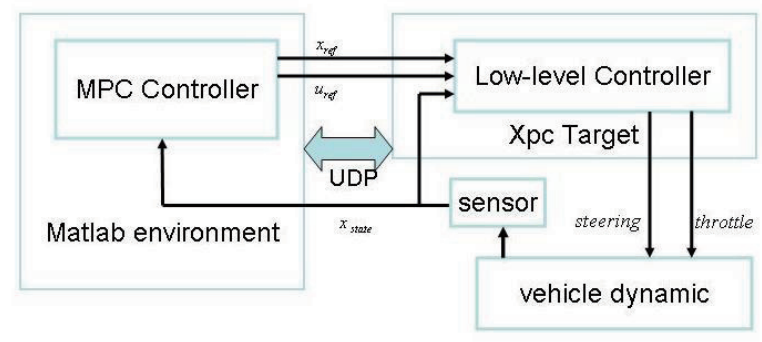

Fig. 6. Experiment implementation structure

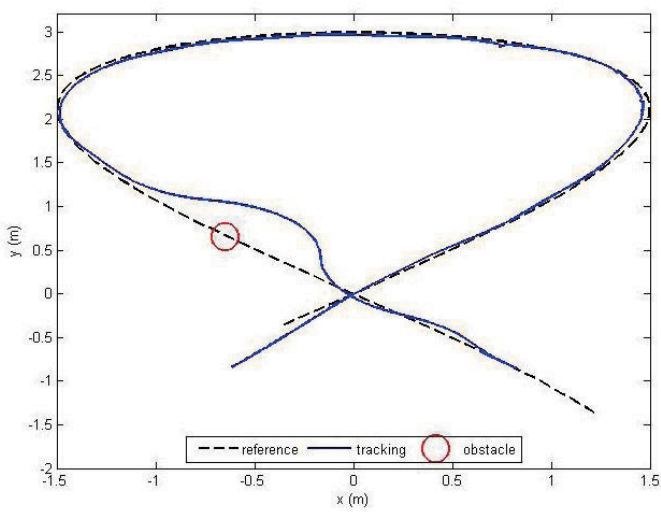

(a) Tracking result
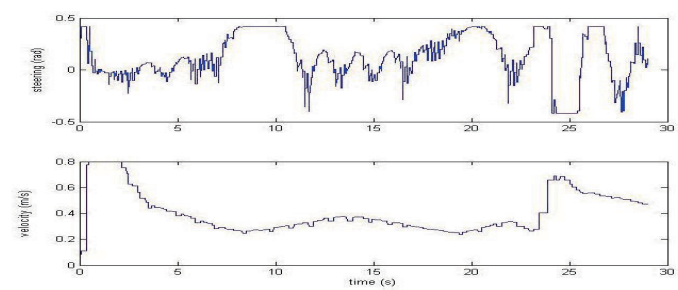

(b) Control signals

Fig. 7. Obstacle avoidance

represent the position of the vehicle when MPC updating the vehicle information. The solid lines evolved from these dots are regenerated local trajectories at each sampling instant.

\section{CONClusion}

This paper describes a two-level control framework for autonomous vehicle trajectory tracking in a dynamic and uncertain environment. The vehicle model, various constraints and real-time information about obstacles and other road users are considered in the local trajectory regeneration by using MPC strategy. The two-level framework overcomes the barriers of implementing computationally intensive MPC on vehicles with fast dynamics, making the MPC strategy more affordable and reliable. The design of MPC and lowlevel tacking control are introduced respectively. Numerical simulation and experiments were carried out to demonstrate the performance of the proposed approach.

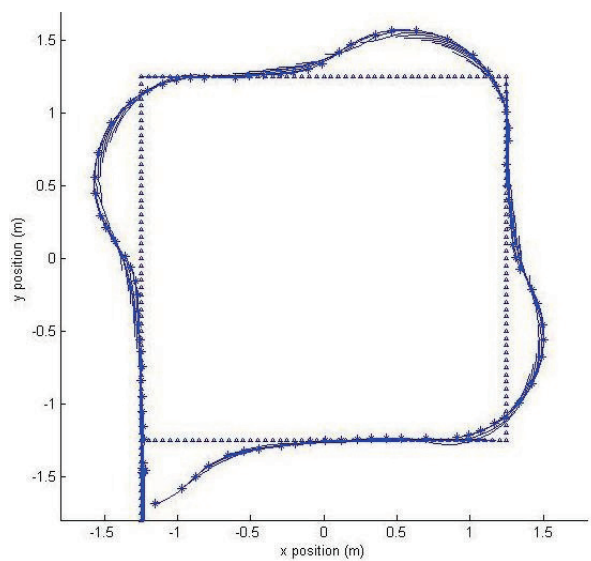

Fig. 8. Square tracking results

\section{REFERENCES}

[1] Y. Kuwata, A. Richards, T. Schouwenaars, and J. How, "Distributed robust receding horizon control for multivehicle guidance," Control Systems Technology, IEEE Transactions on, vol. 15, no. 4, pp. 627-641, July 2007.

[2] P. Morin and C. Samson, "Trajectory tracking for non-holonomic vehicles: overview and case study," in Robot Motion and Control, 2004. RoMoCo'04. Proceedings of the Fourth International Workshop on, 2004, pp. 139-153.

[3] Y. Yoon, J. Shin, H. J. Kim, Y. Park, and S. Sastry, "Model-predictive active steering and obstacle avoidance for autonomous ground vehicles," Control Engineering Practice, vol. 17, no. 7, pp. 741-750, 72009.

[4] A. Ollero and O. Amidi, "Predictive path tracking of mobile robots. application to the cmu navlab," in Advanced Robotics, 1991. 'Robots in Unstructured Environments', 91 ICAR., Fifth International Conference on, 1991, pp. 1081-1086 vol.2.

[5] D. Gu and H. Hu, "Neural predictive control for a car-like mobile robot," Robotics and Autonomous Systems, vol. 39, no. 2, pp. 73-86, 5/31 2002

[6] —- "Receding horizon tracking control of wheeled mobile robots," Control Systems Technology, IEEE Transactions on, vol. 14, no. 4, pp. 743-749, July 2006.

[7] Y. Zhu and U. Ozguner, "Constrained model predictive control for nonholonomic vehicle regulation problem," in Proceedings of the 17th IFAC World Congress, 2008, pp. 9552-9557.

[8] J. Hedrick and Y. Kang, "Linear tracking for a fixed-wing uav using nonlinear model predictive control," Control Systems Technology, IEEE Transactions on, vol. 17, no. 5, pp. 1202-1210, Sept. 2009.

[9] F. Kuhne, W. F. Lages, and J. M. G. da Silva Jr, "Model predictive control of a mobile robot using linearization," in Proceedings of Mechatronics and Robotics, 2004, pp. 525-530.

[10] G. Klancar and I. Skrjanc, "Tracking-error model-based predictive control for mobile robots in real time," Robotics and Autonomous Systems, vol. 55, no. 6, pp. 460-469, 6/30 2007.

[11] K. Kanjanawanishkul and A. Zell, "Path following for an omnidirectional mobile robot based on model predictive control," in Robotics and Automation, 2009. ICRA 'O9. IEEE International Conference on, May 2009, pp. 3341-3346.

[12] H. Kim, D. Shim, and S. Sastry, "Nonlinear model predictive tracking control for rotorcraft-based unmanned aerial vehicles," in American Control Conference, 2002. Proceedings of the 2002, vol. 5, 2002, pp. 3576-3581 vol.5.

[13] D. Q. Mayne, J. B. Rawlings, C. V. Rao, and P. O. M. Scokaert, "Constrained model predictive control: Stability and optimality," Automatica, vol. 36 , no. 6, pp. $789-814,2000$.

[14] E. Gyurkovics and A. M. Elaiw, "Stabilization of sampled-data nonlinear systems by receding horizon control via discrete-time approximations," Automatica, vol. 40, no. 12, pp. 2017 - 2028, 2004.

[15] W. Xi and J. S. Baras, "Mpc based motion control of car-like vehicle swarms," in Control and Automation, 2007. MED'07. Mediterranean Conference on, 2007, pp. 1-6. 